Winter, S. (2019). Performatività e improvvisazione: I'artista Teresa Bandettini Landucci. Italica Wratislaviensia, 10(2), 161-174.

DOI: http://dx.doi.org/10.15804/IW.2019.10.1.23

\author{
Susanne Winter \\ Universität Salzburg \\ susanne.winter@sbg.ac.at
}

\title{
PERFORMATIVITÀ E IMPROVVISAZIONE: L'ARTISTA TERESA BANDETTINI LANDUCCI
}

\author{
PERFORMATIVITY AND IMPROVISATION: \\ THE ARTIST TERESA BANDETTINI LANDUCCI
}

\begin{abstract}
Teresa Bandettini Landucci (1763-1837) was a dancer, poet, and above all amongst the most acclaimed composers of extemporaneous verse in the late $18^{\text {th }}$ century. Yet her legacy is all but forgotten, her name virtually unmentioned in histories of literature. Such an omission is a result of the general dismissal of female writing in literary history; but, in the case of Bandettini, it is all the more pronounced because she was an improviser. The aim of this study is to show that, firstly, in the $18^{\text {th }}$ century, the profession of improvisation was one of the few cultural fields in which women could attain celebrity; and secondly, that the Western emphasis on the written word over orality, on printed documents over ephemeral performances, has meant that improvisation as a performative art has received little recognition or cultural legitimacy.

The art of improvisation experienced a golden age in the second half of the $18^{\text {th }}$ century. This depended upon an expression of enthusiasm within an impromptu performance, characterised by physical presence, the staging of the self, and the involvement of the public. The immediacy of the performance and the authenticity of the artist were decisive elements of this type of performance, though they were rarely recognised within a culture of literacy. Moreover, the transcription and publication of improvised poems could never communicate the nature of these performances and their cultural impact, in part because the oral nature of these texts was considered to be subordinate to meditated poetry. The marginalisation of Bandettini in literary history can therefore be ascribed to the dismissal of categories such as performance, orality, and ephemerality.
\end{abstract}

Keywords: Teresa Bandettini Landucci, improvisation, enthusiasm, performativity, orality 
【1 giudizio espresso nel Dizionario biografico degli italiani su Tere1 sa Bandettini, improvvisatrice tra i più acclamati poeti estemporanei alla fine del Settecento, non potrebbe essere più netto:

La B. fu poetessa di nessuna profondità e sentimento; le sue conoscenze furono assai vaste, ma altrettanto superficiali. Sicura del proprio talento e della propria indiscussa superiorità, la $\mathrm{B}$. non dette mai eccessivo peso alle critiche [...]. Benché molto le giovasse il costante aiuto di molti letterati dell'epoca, primo tra tutti il Bettinelli, che le furono sinceramente amici ed estimatori, essa non ha nulla che la distingua dai numerosi improvvisatori suoi contemporanei [...]. (Scolari Sellerio, 1963, p. 674)

Poetessa di poco valore, pretenziosa, patrocinata da letterati rinomati, improvvisatrice mediocre - un'immagine di donna artista che, secondo Tatiana Crivelli, risulta da quel «meccanismo di selezione e di assunzione» (2003, p. 153) che trasforma un'artista percepita come eccezionale dai contemporanei in un fenomeno al massimo marginale $\mathrm{o}$ addirittura assente nella storia letteraria.

Nel suo saggio del 2003 Crivelli parla del «caso di Teresa», esempio emblematico dell'inadeguata rappresentazione e conservazione della scrittura femminile nella storia letteraria. Tuttavia, il caso di Teresa è un caso particolare che vorrei esaminare più da vicino, concentrandomi su due aspetti a mio avviso strettamente collegati e ugualmente rilevanti nel processo di emarginazione di Teresa Bandettini nella memoria culturale: essere donna ed essere improvvisatrice. Per quest'indagine il secondo fattore, l'improvvisazione, richiede un'apertura del termine di "teatro" verso un concetto più ampio di teatralità e spettacolarità, mettendo l'accento sull'aspetto della "performatività" intesa come peculiarità di un tipo di rappresentazione basata sul corpo e sulla voce davanti a un pubblico fisicamente presente e l'interazione ambivalente di queste componenti ${ }^{1}$.

1 Cfr. Fischer-Lichte, 2000, p. 20: «Performance, die als Vorgang einer Darstellung durch Körper und Stimme vor körperlich anwesenden Zuschauern gefaßt wird und das ambivalente Zusammenspiel aller beteiligten Faktoren beinhaltet». 
Dopo alcuni cenni biografici su Bandettini mi soffermerò quindi sul mestiere di improvvisatrice che le valse non solo una larga notorietà tra i contemporanei ma anche l'ammirazione di autorità letterarie come Alfieri, Bettinelli, Monti, Parini ed altri.

Nata a Lucca nel 1763, Teresa Bandettini esordì come ballerina di teatro, scritturata come prima ballerina a Bastia, Firenze, Bologna, Venezia, prima di cimentarsi come improvvisatrice 2 . Nel 1789 sposò il concittadino e collega-ballerino Pietro Landucci, con cui ebbe quattro figli, di cui tre figlie morte in giovane età, e iniziò la sua carriera di poetessa estemporanea, «compiendo vere e proprie tournées di improvvisazione poetica in molte parti d'Italia» (Ibidem, p. 140). Nel $1794 \mathrm{fu}$ accolta in Arcadia col nome di Amarilli Etrusca ed il suo ritratto, opera di Angelika Kauffmann, fu appeso nel Serbatoio ${ }^{3}$. Si esibì a Bologna, a Venezia, a Udine, a Ferrara, a Padova, a Roma, a Firenze e altrove; conobbe personaggi autorevoli che la introdussero nella società letteraria e si creò una rete di contatti importanti. Tenne numerose accademie di improvvisazione e acquistò fama, però nessuna rendita con la quale poter vivere tranquillamente. Neanche le speranze riposte nell'invito alla corte di Vienna si realizzarono. Due anni dopo rientrò in Italia, si ritirò dalle scene e ottenne infine un vitalizio - prima dal duca di Modena, poi dalla duchessa di Lucca che le permise alla fine della sua vita di far ritorno nella città natale. Quantunque il suo prestigio si basi soprattutto sull'attività di improvvisatrice, Teresa Bandettini «può vantare secondo Tatiana Crivelli - una bibliografia di tutto rispetto» (Ibidem, pp. 146-148). Si contano sei edizioni di rime, fu inoltre traduttrice letteraria, giornalista, scrittrice di tragedie, di poemi didascalici, di libretti per musica e di un poema epico in venti canti, La Teseide. Morì a Lucca

2 La maggior parte delle informazioni sulla vita di Teresa Bandettini provengono dalla sua autobiografia datata 1825 e pubblicata in Di Ricco, 1990, pp. 229-246 e dalle Memorie per servire all'Istoria poetica di Amarilli Etrusca di Tommaso Trenta, scritte intorno al 1800 e pubblicate in Caspani Menghini, 2011, pp. 45-60. Un importante studio sulla vita e la parte improvvisatoria dell'opera di Teresa Bandettini è di Vannuccini, 1899, pp. 501-526, 732-756.

3 Il Serbatoio è la sede dell'archivio con la sala per le adunanze amministrative dell'Arcadia. 
all'età di 73 anni, fu celebrata con onori straordinari dall'Accademia Lucchese lo stesso anno e poi quasi dimenticata.

Ai suoi tempi, la notorietà di Teresa Bandettini nacque e crebbe sulla base della sua «facil vena d'improvvisare» (Di Ricco, 1990, p. 239) come scrive nella sua breve autobiografia - e delle sue performances improvvisatorie nei salotti e nelle accademie. È però proprio questo tipo di genere artistico e manifestazione culturale che durante l'Ottocento perse in prestigio e a poco a poco scomparve tanto che a metà Novecento Benedetto Croce può parlare dell'improvvisazione come «evento storico», un evento cioè ben delimitato nel tempo e nello spazio, di poca rilevanza fuori di questi confini. Secondo lui gli improvvisatori «hanno il loro ufficio e il loro significato in un paese e in una età determinata, in Italia, tra l'Arcadia settecentesca e l'Arcadia romantica» (1949, p. 299). È infatti nella seconda metà del Settecento, che l'improvvisazione visse la sua età aurea diventando pratica arcadica. La poesia estemporanea, a partire da una forma di competizione giocosa, si trasformò progressivamente in moda diffusa nelle accademie e divenne divertimento occasionale per letterati di rango - sia uomini che donne - oppure passatempo popolare. Il concetto estetico sotteso a questa moda è "l'entusiasmo", l'ispirazione, il genio che si materializza nel «poetare rapido e immediato o (...) all'improvviso» (Ibidem, p. 301) e non è un caso che, per spiegare il "funzionamento" dell'entusiasmo nell'opera dallo stesso titolo, Dell'entusiasmo delle belle arti (1769), Bettinelli scelga l'esempio dell'abate Bartolomeo Lorenzi (1732-1822), padre veronese noto per le sue qualità d'improvvisatore, e descriva dettagliatamente una performance estemporanea - descrizione alla quale riandremo in seguito. Anche se è incontestabile che l'improvvisazione raggiunse l'apice alla fine del Settecento, in realtà si trattava di una pratica esercitata in Italia da secoli, come dimostra il saggio Über die Improvisatoren (1806) di Karl Ludwig Fernow, testimone diretto del fenomeno improvvisatorio durante il suo soggiorno a Roma negli anni novanta del Settecento, ed è dunque difficile classificarla e minimizzarla come «evento storico» e quindi fenomeno trascurabile.

Il giudizio negativo sopracitato e la quasi assenza dell'improvvisazione e degli improvvisatori nella memoria culturale non sono sola- 
mente riconducibili al cambiamento dell'estetica nell'Otto/Novecento e al conseguente eclissarsi di questo genere artistico, bisogna invece tenere conto anche di altri aspetti per spiegare la situazione e mi pare che proprio la dicotomia oralità/scrittura e il concetto di "performatività" possano avere un impatto notevole sulla valutazione della poesia estemporanea nonché degli improvvisatori e delle improvvisatrici.

Considerando per primo l'aspetto della performatività, possiamo constatare che l'improvvisazione come esercizio socio-mondano assomiglia molto a quello che oggi chiameremmo performance. L'insistenza sulla presenza fisica, la messa in scena del proprio io e il coinvolgimento emotivo del pubblico sono gli elementi più importanti ${ }^{4}$ che appaiono chiaramente sia nella descrizione dell'Abate Lorenzi in Dell'entusiasmo di Bettinelli, sia nelle osservazioni di Fernow.

Per Bettinelli è quello «che chiamasi Furor poetico, Ispirazion, Rapimento, in una parola Entusiasmo, ovver Estro (...) che contraddistingue propriamente i veri poeti, pittori, oratori, e compagni loro nelle arti, constituendo l'indole loro, e talento in una classe particolare» e «non v'ha forse esempio più manifesto dell'Entusiasmo quasi visibile quanto nell'occasione d'improvvisare» (1769, pp. 7, 48). L'improvvisazione è quindi quell'entusiasmo in actu che Bettinelli illustra sull'esempio dell' «eccellente Poeta estemporaneo», Abate Lorenzi. Suddividendo la performance in sei fasi, dall'esordio ovvero «elevazione», passando per visione, rapidità, spettacolo, passione, fino alla trasformazione o trasfusione, Bettinelli evoca lo spettacolo prestando particolare attenzione alla fisicità dell'improvvisatore e all'interazione con il pubblico (Ibidem, pp. 46-47, 142).

L'esibizione segue non solo la drammaturgia dell'ispirazione descritta da Bettinelli, ma anche una drammaturgia teatrale nell'apparizione dell'improvvisatrice o dell'improvvisatore davanti al pubblico con saluti, complimenti, congedi, ringraziamenti, epiloghi o formule impiegate per interrompere l'improvvisazione e introdurre una pausa (Di Ricco, 1990, pp. 167-168; Fernow, 1806, pp. 311-318), frequentemente riempita dalla musica eseguita sullo strumento accompagnatore

${ }^{4}$ Cfr. Pavis, 1998, voce "performance" e "performer". 
dell'improvvisatrice/improvvisatore. È evidente che al centro della performance sta l'improvvisazione stessa sopra temi proposti dal pubblico, spesso di carattere mitologico, religioso o politico, ma è altrettanto importante che questa sia incorniciata da parti introduttive e conclusive. Fernow descrive come all'inizio dello spettacolo, durante la disposizione dell'improvvisazione, è il musicista a divertire gli astanti in attesa della performance vera e propria, che sembra elevare l'artista a uno stato di rapimento geniale, dopodiché la prestazione si chiude con un ingegnoso riassunto di tutti i temi improvvisati ${ }^{5}$.

L'aspetto spettacolare dell'arte improvvisatoria mi sembra decisivo per una corretta valutazione sia dell'improvvisatrice/dell'improvvisatore, sia delle reazioni dei contemporanei, sia del suo status nella storia letteraria. In tutte le descrizioni contemporanee, la presenza scenica dell'artista è centrale, la sua bravura intellettuale, gli indizi dell'entusiasmo, si manifestano anche in modo corporeo. Scrive Bettinelli:

5 Nel resoconto di un'accademia improvvisatoria Fernow utilizza parole appartenenti chiaramente al vocabolario teatrale: «Ein Akt des Schauspiels ist nun geendigt. Der Sänger erholt sich, troknet den Schweis von der glühenden Stirne, und zerstreut sich auf wenige Minuten durch die Unterhaltung mit der ihn froh umringenden Schar» (Fernow, 1806, pp. 313-314): «È finito il primo atto dello spettacolo. Il cantante si riposa, asciuga il sudore sulla fronte accaldata e si rilassa conversando un attimo con il pubblico che lo attornia festevole» (traduzione mia). Può sorprendere il termine "cantante", ma c'è una componente musicale nell'improvvisazione - non ancora tutta chiarita (cfr. Di Ricco, 1990, pp. 22-25) - non solo riguardo al virtuosismo metrico ma anche relativamente all'esecuzione parlata o cantata e l'accompagnamento. Mentre Fernow menziona la musica come elemento accessorio in tutte le improvvisazioni alle quali ha assistito, nei testi di Teresa Bandettini manca ogni accenno alla musica, anche se esiste almeno una testimonianza di un'improvvisazione di Bandettini con accompagnamento musicale a Roma, anche se lo strumento non è precisato (Crivelli, 2003, p. 144), e una di un'adunanza all'Accademia degli Oscuri a Lucca durante la quale fu accompagnata da un accademico al pianoforte (Caspani Menghini, 2011, pp. 89 e 484); visto che l'evento è datato 1794 è probabile che si tratti di un pianoforte a martelli o piuttosto di un clavicembalo. Secondo Fernow, molti improvvisatori e improvvisatrici inventano una melodia specifica per ogni metro, per abbellire la recitazione e per facilitarla, e ne pubblica alcuni esempi alla fine del suo libro (Fernow, 1806, pp. 315-316 e ultime pagine del libro). 
Tu vedi ancor nell'esterno gl'indizj di sua partenza. Un serenarsi la faccia, un guardar alto, ed astratto da tutti gli oggetti presenti, una immobilità del corpo come abbandonato, o dimenticato, ch'egli non è più dov'era. [...] Già si fissa, già vede qualche cosa, che non vedeva [...] Poco a poco vi si affeziona, e gode, e gusta, e il fuoco della passione a quelle viste mirabili, a quelle nobili attrattive s'accende, e cresce, e serpe in ogni sua vena, onde gli occhi ardono, le guance arrossano, sorride la bocca, giubila, freme, e commovesi la persona ancor non di rado.

Ed è ben viva fiamma nel vero, ed ardente, che il comprende, e trasporta, sicchè più s'alza la voce, affrettasi il gesto, e vibransi i moti, e sopra tutto un'affluenza, un affollamento di concetti, di rime, d'immagini ne trabocca, che le parole non bastano, e vanno tarde, e s'affanna, e s'ingorga, e sembrano i versi sospingersi, accavallarsi, inondare, precipitare. [...] Due singolari osservazioni possono farsi al finire 1'azione, l'una sopra il Poeta, l'altra su i circostanti, che possono dar nuovo lume. Ed è in prima lo spossamento di forze nell'Improvvisatore per l'esercizio violento, ed impetuoso, quasi sopra la natural attitudine, ed uso degli organi; poi negli uditori un silenzio... (1769, pp. 49-51)

L'immediatezza dell'esibizione e l'autenticità dell'artista sono elementi indispensabili a questo tipo di prestazione, e la presenza fisica è intimamente legata al virtuosismo intellettuale. Anche Fernow sottolinea la grazia delle sembianze e delle movenze di Teresa Bandettini improvvisatrice: «un paio di grandi occhi ardenti, nei quali si specchia ogni stato d'animo le conferiscono durante il canto l'aspetto di un'appassionata sacerdotessa di Apollo» (1806, p. 388) ${ }^{6}$. Nel «caso di Teresa» possiamo inoltre supporre che durante la sua carriera di prima ballerina abbia perfezionato l'espressività e l'armonia dei suoi gesti e movimenti ${ }^{7}$.

Per di più, la performance improvvisatoria è caratterizzata da un'intensa interazione tra improvvisatrice/improvvisatore e pubblico. Non

${ }^{6}$ «Ihr Vortrag bezaubert durch seine Annehmlichkeit, und durch die Grazie ihres Anstandes; diese, und ein grosses feuriges Augenpar, in dem jede Stimmung des Gemüths sich abspiegelt, geben ihr wärend des Gesanges das Ansehen einer begeisterten Priesterin des Apollo».

7 Per questo mi sembra poco probabile che avesse «gesti e moti spesse volte incomposti e ridicoli» (Vannuccini, 1899, p. 737). 
solo le proposte per il tema dell'improvvisazione vengono dal pubblico, ma anche l'improvvisazione stessa vive del reciproco influsso per il quale Bettinelli usa l'immagine del gioco a palla: l'entusiasmo dell'artista si trasmette al pubblico, ed è «quasi come un mandare, e rimandare di palla il riverberarsi dell'Estro, e il ripercotersi dal Poeta agli uditori, e da loro a lui crescendo sempre a vicenda in entrambi il godimento, il rapimento, l'ebrietà» (Bettinelli, 1769, p. 51).Tuttavia, questa interazione non è automatica; dipende dalla disposizione dell'artista nonché dalla composizione del pubblico, poiché «assai giova una scelta corona d'ascoltatori, e meglio se sono amici, e pregiati da lui [i.e. l'improvvisatore]» (Ibidem, p. 52). Per le serate improvvisatorie, si possono distinguere due ambiti con due tipi di pubblico diversi: quelle private nei salotti e nelle case dei nobili e quelle pubbliche nelle accademie dove si improvvisava dietro pagamento di una somma ${ }^{8}$. Più l'artista e il pubblico concordano, più si stimolano reciprocamente fino a un'estasi collettiva, di modo che lo spettatore esce da questo spettacolo «pazzo, fanatico, sorpreso al segno del delirio» (Vannuccini, 1899, pp. 517-18) ${ }^{9}$, come scrive un fiorentino in una lettera che si riferisce a una serata improvvisatoria a Firenze nel 1794, degna di nota perché riunì due improvvisatrici tra le più famose, Teresa Bandettini e la Fantastici (i.e. Fortunata Sulgher), davanti a un pubblico di 140 o 150 persone, tutte avide di vedere e sentire le improvvisatrici. In questa determinata circostanza, oltre l'aspetto emotivo non è da sottovalutare quello competitivo, poiché le due donne improvvisarono in dialogo sul tema di Ero e Leandro.

Per il nostro «caso di Teresa», l'improvvisazione, in quanto esecuzione dal vivo con gli aspetti di presenza scenica dell'artista, di realizzazione immediata e messa in scena della propria inventiva nonché di interazione con il pubblico, è significativa in due aspetti, uno attinente alla sua specificità come arte performativa, l'altro inerente all'oralità.

${ }^{8}$ In una lettera a Bettinelli (21 agosto 1801) Teresa Bandettini distingue chiaramente tra accademia pubblica e privata: «voglio aver riguardo alla mia salute essendo di maggior impegno una accademia pubblica che una privata» (Bandella, 2006, p. 110).

9 Ripreso da Di Ricco, 1990, p. 15. 
Il mestiere di improvvisatore/improvvisatrice si inserisce in un quadro socio-mondano di fine Settecento caratterizzato dall'estendersi degli attanti, dei partecipanti e del pubblico (Habermas, 1977, p. 53) e da un'ampia sociabilità e visibilità dei fenomeni letterari nei salotti, nelle accademie, nel giornalismo. Un ruolo importante in questo contesto spetta alle donne che colgono l'occasione di partecipare e di mettersi in scena, trattandosi di un campo letterario nuovo, ancora aperto e poco definito ${ }^{10}$. L'improvvisazione è pertanto un mestiere praticato da uomini e donne ugualmente, ed è interessante che le descrizioni e i resoconti di Fernow delle performance improvvisatorie contemporanee siano ripartiti parimenti tra improvvisatrici e improvvisatori e che i criteri di valutazione non differiscano. Acquistare celebrità come improvvisatrice è altrettanto possibile come raggiungere la notorietà come attrice o musicista (strumentalista), mentre sembra quasi impossibile o comunque molto difficile diventare una scrittrice o compositrice affermata. Sono soprattutto le arti performative quelle cui le donne hanno diritto di accesso e in cui possono brillare, mentre l'ammissione nel campo letterario e musicale, prettamente maschile, sembra ostacolata per via dell'educazione, dei pregiudizi ecc.

Se è vero che le donne non sono svantaggiate in questo campo artistico, tuttavia, e purtroppo, la celebrità nelle arti performative è poco durevole, trattandosi di fenomeni effimeri, impossibili da perpetuare, almeno in quell'epoca. Ora, siccome le storie della letteratura, del teatro e della musica si fondano di solito su documenti stampati o almeno scritti, i fenomeni effimeri, tra cui tutte le varietà di performances, vengono facilmente emarginati o dimenticati. Anche se il fenomeno colpisce indifferentemente artisti performativi di sesso maschile e femminile, sono le donne a soffrirne in più larga misura, essendo uno dei pochi campi culturali nei quali possono emergere e dimostrare la parità.

A questo meccanismo inerente alla dicotomia dell'effimero e del duraturo, si aggiunge un altro aspetto, quello della gerarchia nel dualismo di oralità e scrittura. L'oralità della performance non è solo un fenomeno effimero, è un fenomeno subalterno, visto che l'idea della letteratura

${ }^{10}$ Per un quadro più ampio vedi Graziosi, 1992. 
focalizzata sulla scrittura, e quindi la priorità dello scritto sull'oralità, è fortemente radicata nella cultura occidentale.

Teresa Bandettini sembra avere piena coscienza di questo fatto e lotta su due fronti: da una parte tenta di cimentarsi come scrittrice, dall'altra palesa riserve riguardo alla stampa dei suoi versi improvvisati perché non rispondenti alle aspettative nei confronti dello scritto.

Non a caso, nella sua breve autobiografia, Teresa Bandettini fa corrispondere l'inizio della sua carriera di poetessa e l'abbandono di quella di ballerina e «donna di teatro» con l'ingresso in alcune accademie e la pubblicazione del suo «poemetto della Morte d'Adone» (Di Ricco, 1990, pp. 240, 244). Mentre le accademie costituirono la base per una necessaria rete di contatti che Bandettini coltivò e mantenne per tutta la vita, assicurandosi così sostegno e pubblicità per le sue tournées d'improvvisatrice ${ }^{11}$, il poemetto in quattro canti in ottava rima, stampato nel 1790 a spese del Senatore Lodovico Savioli di Bologna, le avrebbe dovuto aprire le porte nel campo della letteratura scritta. Della raccolta di poesie giovanili e improvvisate (Ibidem, p. 242), uscita quattro anni prima in due tomi, si dichiarava invece scontenta distinguendo così chiaramente tra testi concepiti fin dall'inizio per la stampa e le poesie occasionali. Conseguentemente accanto alla carriera d'improvvisatrice continuò a impegnarsi nella letteratura scritta con due tragedie, Il Polidoro, edita nel 1794, e Rosmunda in Ravenna (1827), con poesie, odi, un'azione drammatica, La caduta dei giganti (1814) e soprattutto con il poema La Teseide, stampato nel 1805 dopo una lunga fase di elaborazione.

Nella corrispondenza con Bettinelli, che assomma a circa 200 lettere (Bandella, 2006, p. 458), si parla di quest'opera dal 1799 fino all'anno della pubblicazione, ed è significativo come quest'amicizia epistolare dimostri una crescente sicurezza di sé da parte della scrittrice (Ibidem, pp. 461-462). Se all'inizio sono un maestro e un'allieva, il dotto e il talento naturale a scambiarsi lettere, nel 1805 è la scrittrice che si rivolge al letterato. In una lettera del 1799 Bandettini scrive:

11 Cfr. Monica Bandella che parla di una «massiccia presenza di nomi di letterati noti» nella corrispondenza con Bettinelli (2006, p. 423). 
Io vi voglio giudice severo. Taccia l'amico, il maestro indulgente, avvertitemi ove io abbia mancato acciocché sappia correggermi per l'avvenire, lodatemi in quei passi che io ho messi sicuri ond'io non perda il coraggio, che l'avvilimento sarebbe il peggio di tutto e mi renderebbe incerta e diffidente di me medesima. Da voi attendo questi lumi. (Bandella, 2006, p. 48) ${ }^{12}$

Sei anni dopo, in occasione della pubblicazione di La Teseide, l'autrice riferisce a Bettinelli le lodi di un terzo, Giuseppe Maria Pagnini (Ibidem, p. 328), responsabile della stampa del poema: «Pagnini mi scrive che l'edizione dell'opera mia va avanti con la maggior sollecitudine (...). Il vostro Poema quanto più l'esamino tanto più mi sodisfa (...) La singolare stima che ho sempre avuta del vostro maraviglioso talento si è accresciuta a dismisura per una produzione sì grande sì ben condotta, e sì piena d'eloquenza poetica qual è la vostra Teseide» (Ibidem, p. 194) ${ }^{13}$. A luglio, infine, chiede a Bettinelli di occuparsi della distribuzione dei volumi a Mantova e di ritirarne i denari, concludendo «Che ne dite mio rispettabil Diodoro lo farete sì, o no?» (Ibidem, p. 198) ${ }^{14}$. La crescente autonomia di Bandettini nel campo della letteratura scritta si vede anche nel fatto che, a differenza delle opere precedenti, non sono le parole di qualche illustre personalità ad introdurre La Teseide ed assicurarne la validità, ma è l'autrice stessa a presentare il suo poema.

Peraltro, sappiamo che tutti questi sforzi si rivelarono poco efficaci per entrare nella storia letteraria e nella memoria culturale, giacché, come abbiamo visto, per le donne di quest'epoca era difficile conquistare una posizione importante nel campo letterario.

D'altra parte, immortalarsi come improvvisatrice pubblicando le poesie estemporanee significò far fronte alla problematica dicotomia oralità/scrittura. Sia il desiderio degl'improvvisatori di perpetuare la celebrità che l'entusiasmo e il fanatismo del pubblico finirono col promuovere la trascrizione e la stampa di versi improvvisati. Tuttavia, Bandettini è consapevole del rischio insito nella trasformazione dell'orale in scritto. Da un lato gli estemporanei stampati fanno pubblicità

\footnotetext{
12 Lettera del 3 novembre 1799.

13 Lettera del 14 aprile 1805.

14 Lettera del 21 luglio 1805.
} 
e documentano il successo dell'improvvisatrice o dell'improvvisatore, dall'altro, in questa forma fissata e durevole, si prestano al confronto con le poesie meditate - un confronto sicuramente poco vantaggioso per gl'improvvisi. L'ambiguità di tale impresa è comprovata dalla vicenda di un progetto avviato da Tommaso Trenta, segretario dell'Accademia degli Oscuri di Lucca e poeta arcade, consistente in una raccolta dei versi improvvisati di Teresa Bandettini. Questo progetto, analizzato da Tatiana Crivelli e successivamente pubblicato e ampiamente commentato da Franca Caspani Menghini (Crivelli, 2003; Caspani Menghini, 2011), ebbe inizio nel 1794, dopo un soggiorno dell'improvvisatrice a Lucca, dove aveva lasciato un'impressione e un fascino profondi, ma rimase incompiuto a causa della riluttanza della poetessa stessa, che solo due anni prima della morte si decise a pubblicare una raccolta complessiva delle sue poesie estemporanee, approntata però da un altro ammiratore (Crivelli, 2003, p. 168).

La preoccupazione di Teresa Bandettini relativamente alla pubblicazione delle sue poesie improvvise mi pare considerevole sotto vari punti di vista: è ovvio che faccia parte di una strategia di autopromozione e di autostilizzazione, per quanto ambigua, perché da una parte mira alla documentazione e alla diffusione della propria produzione poetica improvvisa, dall'altra cerca di evitare un confronto qualitativo con la poesia scritta. Una volta avulse dal contesto performativo dell'improvvisazione, le parole devono fare a meno della compresenza fisica dell'artista e del pubblico, della stimolazione reciproca, della componente visiva, dell'immediatezza e dell'effimero, e si riducono a semplici segni neri sulla pagina bianca esposti nudi e crudi alla lettura. È altrettanto evidente che il virtuosismo improvvisatorio, oggidì quasi inconcepibile, va di pari passo con una certa riduzione, banalizzazione e stereotipia mal viste nella poesia meditata. Nel contesto performativo, invece, questi criteri non sono decisivi per il successo, che si fonda soprattutto sul carattere evenemenziale dell'esibizione, sull'esaltazione emotiva o - per usare il termine bettinelliano - sull'entusiasmo dell'artista e del pubblico.

La stampa di poesie estemporanee solleva inoltre un'ulteriore questione riguardo all'attendibilità e paternità/maternità del testo. Visto che per lo più non erano gli improvvisatori o le improvvisatrici che mette- 
vano per iscritto le loro invenzioni, ma gli ascoltatori che trascrivevano le poesie durante la declamazione e visto che esisteva una vera e propria «caccia alle trascrizioni» (Di Ricco, 1990, p. 35) ci si può chiedere quanto fedeli potessero essere all'esecuzione dal vivo. Non sappiamo, per esempio, se il riserbo di Teresa Bandettini su quel progetto di pubblicazione dei suoi estemporanei iniziato da Trenta sia dovuto anche a questo aspetto oltre che alla preoccupazione in generale sulla trasformazione dall'oralità alla scrittura.

Per concludere possiamo constatare che «la degenerazione del prestigio della poetessa lucchese» (Crivelli, 2003, p. 150) e l'emarginazione di Teresa Bandettini non possono essere ricondotte soltanto alla rappresentanza inadeguata della scrittura femminile nella storia letteraria, ma vanno attribuite in larga misura al carattere performativo, orale ed effimero delle sue prestazioni improvvisatorie, valorizzanti la processualità, la corporeità nonché l'interazione immediata fra artista e pubblico. Che sia poi nel campo delle arti performative, caratterizzate da una certa parità tra $\mathrm{i}$ generi, dove le donne poterono affermarsi ed arrivare alla celebrità e non nel campo proprio della letteratura, questo è un altro problema.

\section{BIBLIOGRAFIA}

Bandella, M. (2006). „, Tu al difficil sentier di gloria il varco / mi apristi, e tu la man tremante in pria / ferma rendesti ad incurvar grand'arco ". Il carteggio tra Saverio Bettinelli e Teresa Bandettini Landucci (1793-1808) (Ph.D. Thesis). Università di Salisburgo.

Bettinelli, S. (1769). Dell'entusiasmo delle belle arti. Milano: Giuseppe Galeazzi.

Caspani Menghini, F. (2011). L'estro di Amarilli e la tenacia di Artinio. Poesie estemporanee di Teresa Bandettini raccolte dal concittadino Tommaso Trenta (1794-1799). Lucca: Accademia Lucchese di Scienze, Lettere e Arti.

Crivelli, T. (2003). Le memorie smarrite di Amarilli. Versants, 46 (Littérature au féminin, a cura di G. Codoue, T. Crivelli, Y. Foehr-Janssons), 139-190.

Croce, B. (1949). Gl'improvvisatori. In Idem, La letteratura italiana del Settecento (pp. 299-311). Bari: Laterza. 
Di Ricco, A. (1990). L’inutile e maraviglioso mestiere. Poeti improvvisatori di fine Settecento. Milano: FrancoAngeli.

Fernow, C.L. (1806). Über die Improvisatoren. In Idem, Römische Studien II (295-416). Zürich: Gessner.

Fischer-Lichte, E. (2000). Theatralität und Inszenierung. In E. Fischer-Lichte \& I. Pflug (Eds.), Inszenierung und Authentizität (pp. 11-27). Tübingen, Basel: Francke.

Graziosi, E. (1992). Arcadia femminile: presenze e modelli. Filologia e Critica, XVII (3), 321-358.

Habermas, J. (1977). Storia e critica dell'opinione pubblica. Bari: Laterza.

Pavis, P. (1998). Dizionario del teatro, ed. italiana a cura di Paolo Bosisio. Bologna: Zanichelli.

Scolari Sellerio, A. (1963). Bandettini, Teresa (Amarilli Etrusca). In Dizionario Biografico degli Italiani, vol. 5. Roma: Istituto dell'Enciclopedia Italiana. Retrieved from http://www.treccani.it/enciclopedia/teresa-bandettini_(Dizionario-Biografico)

Vannuccini, G. (1899). Una poetessa improvvisatrice della seconda metà del secolo XVIII. Rassegna nazionale CVIII, 501-526; 732-756.

\begin{abstract}
Riassunto: Teresa Bandettini (1763-1837) è stata una ballerina e poetessa e soprattutto un'improvvisatrice tra i più acclamati poeti estemporanei alla fine del Settecento. Della gloria di quest'epoca, nella memoria culturale odierna non resta alcuna traccia, e il nome della poetessa improvvisatrice è quasi sparito dalle storie letterarie. A spiegare questa degenerazione del prestigio si può certamente addurre l'inadeguata rappresentazione della scrittura femminile nella memoria storica in generale, ma nel caso specifico di Teresa Bandettini sono due aspetti strettamente collegati tra di loro che hanno portato all'emarginazione dell'artista: essere donna ed essere improvvisatrice.

Scopo della presente indagine è di mostrare che da un lato, nel Settecento, il mestiere dell'improvvisazione è uno dei pochi campi culturali nei quali anche le donne possono raggiungere celebrità e notorietà, mentre dall'altro l'improvvisazione come arte performativa non è mai entrata nella memoria culturale occidentale, fondata sulla priorità dello scritto sull'oralità, su documenti stampati e non su fenomeni effimeri, tra cui tutte le varietà di performances.

L'improvvisazione, che visse la sua età aurea nella seconda metà del Settecento in rapporto con il concetto dell'entusiasmo è una performance estemporanea caratterizzata dalla presenza fisica, dalla messa in scena del proprio io e dal coinvolgimento del pubblico. L'immediatezza dell'esibizione e l'autenticità dell'artista sono elementi decisivi in questo tipo di prestazione, e tuttavia poco rilevanti per una cultura dello scritto e del duraturo. L'eventuale trascrizione e pubblicazione delle poesie improvvisate non riescono a rimediare alla situazione, perché il carattere orale dei testi è percepito come fenomeno subalterno rispetto alla poesia meditata. L'emarginazione nella storia letteraria della a suo tempo famosissima improvvisatrice Teresa Bandettini può dunque essere ricondotta alla scarsa valorizzazione delle categorie del performativo, dell'orale e dell'effimero.
\end{abstract}

Parole chiave: Teresa Bandettini Landucci, improvvisazione, entusiasmo, performatività, oralità 